\title{
Erratum
}

\section{Integrated spectrum of the planetary nebula NGC 7027}

\author{
Y. Zhang ${ }^{1, \star, \star \star}$, X.-W. Liu ${ }^{1}$, S.-G. Luo ${ }^{1}$, D. Péquignot ${ }^{2}$, and M. J. Barlow ${ }^{3}$ \\ 1 Department of Astronomy, Peking University, Beijing 100871, PR China \\ e-mail: zhangy96@hku.hk \\ 2 LUTH, Laboratoire l'Univers et ses Théories, associé au CNRS (FRE 2462) et à l'Université Paris 7, Observatoire de Paris-Meudon, \\ 92195 Meudon Cedex, France \\ 3 Department of Physics and Astronomy, University College London, Gower Street, London WC1E 6BT, UK
}

A\&A 442, 249-262 (2005), DOI: 10.1051/0004-6361:20052869

Key words. line: identification - ISM: abundances - planetary nebulae: individual: NGC 7027 - Errata, addenda

In Table 5, the ID numbers should be as follows:

Table 5. Plasma diagnostics.

\begin{tabular}{|c|c|c|}
\hline$\overline{\mathrm{ID}}$ & Diagnostic & $\overline{\text { Result }}$ \\
\hline & & $T_{\mathrm{e}}(\mathrm{K})$ \\
\hline 1 & {$[\mathrm{Ne}$ III $] 15 \mu \mathrm{m} /(\lambda 3868+\lambda 3967)$} & 12900 \\
\hline 2 & [Ar III] $\lambda 7135 / \lambda 5192$ & 12800 \\
\hline 3 & {$[\mathrm{O}$ III $](\lambda 4959+\lambda 5007) / \lambda 4363$} & 12600 \\
\hline 4 & {$[\mathrm{~N}$ II $](\lambda 6548+\lambda 6584) / \lambda 5754$} & 12900 \\
\hline 5 & {$[\mathrm{Ne}$ IV] $\lambda 1602 /(\lambda 2422+\lambda 2425)$} & 15300 \\
\hline 6 & [O III] $\lambda 4363 / \lambda 1663$ & $\gtrsim 14700$ \\
\hline 7 & {$[\mathrm{O} \mathrm{II}](\lambda 7320+\lambda 7330) / \lambda 3726$} & 18700 \\
\hline 8 & {$[\mathrm{O} \mathrm{I}] \lambda 5577 /(\lambda 6300+\lambda 6363)$} & 10400 \\
\hline \multirow[t]{6}{*}{9} & {$[\mathrm{Mg} \mathrm{v}] \lambda 2783 / 5.6 \mu \mathrm{m}$} & 15600 \\
\hline & Не I $\lambda 6678 / \lambda 4471$ & 9430 \\
\hline & He I $\lambda 6678 / \lambda 5876$ & 12700 \\
\hline & He I $\lambda 7281 / \lambda 5876$ & 8800 \\
\hline & He I $\lambda 7281 / \lambda 6678$ & 10530 \\
\hline & $\mathrm{BJ} / \mathrm{H} 11$ & 12800 \\
\hline & & $\log N_{\mathrm{e}}\left(\mathrm{cm}^{-3}\right)$ \\
\hline 10 & {$[\mathrm{O}$ II] $\lambda 33726 / \lambda 3729$} & 4.68 \\
\hline 11 & {$[\mathrm{Ar}$ IV] $\lambda 4740 / \lambda 4711$} & 4.77 \\
\hline 12 & [C III] $\lambda 1906 / \lambda 1909$ & 4.80 \\
\hline 13 & {$[\mathrm{Cl} \mathrm{III]} \lambda 5537 / \lambda 5517$} & 4.70 \\
\hline 14 & {$[\mathrm{Ne}$ IV] $\lambda 2425 / \lambda 2423$} & 4.69 \\
\hline 15 & [S II] $\lambda 6731 / \lambda 6716$ & $\gtrsim 5$ \\
\hline 16 & [Si III] $\lambda 1884 / \lambda 1892$ & 4.54 \\
\hline 17 & {$[\mathrm{Ne} \mathrm{v}] 24 \mu \mathrm{m} / 14 \mu \mathrm{m}$} & 4.31 \\
\hline 18 & {$[\mathrm{Ne}$ III $] 15 \mu \mathrm{m} / 36 \mu \mathrm{m}$} & 4.89 \\
\hline 19 & {$[\mathrm{Fe}$ III] $\lambda 4881 / \lambda 4701$} & 5.27 \\
\hline 20 & {$[\mathrm{Fe}$ III] $\lambda 4701 / \lambda 4733$} & 4.50 \\
\hline \multirow[t]{3}{*}{21} & [Fe III] $\lambda 4733 / \lambda 4754$ & 4.27 \\
\hline & H I Balmer decrement & $\sim 5$ \\
\hline & He II Pfund decrement & $\sim 5$ \\
\hline
\end{tabular}

* Also Space Telescope Science Institute, 3700 San Martin Drive, Baltimore, MD 21218, USA.

$\star \star$ Current address: Department of Physics, University of Hong Kong, Hong Kong, PR China. 